\title{
Investigation of $\beta$ defensin gene expression in the ocular anterior segment by semiquantitative RT-PCR
}

\author{
Ordan J Lehmann, Imran R Hussain, Peter J Watt
}

\begin{abstract}
Aim-To determine if $\beta$ defensins are expressed in the anterior segment of the eye and to determine the temporal pattern of expression using a real time semiquantitative reverse transcription polymerase chain reaction (RT-PCR).

Methods-Ocular tissue (corneal epithelium, conjunctiva, iris, and lens capsule) was collected from 23 patients undergoing surgery. Serial corneal or conjunctival impression cytology was performed on a separate group of 10 patients undergoing corneal tunnel phacoemulsification or trabeculectomy. The samples were analysed for $\beta$ defensin mRNA by semiquantitative RT-PCR and the MRNA standardised for cell numbers.

Results-RT-PCR amplified $\beta$ defensin 1 mRNA from all lens capsule (six) and corneal (five) samples and all but one of the conjunctival (six) and iris samples (six). $\beta$ Defensin 2 mRNA was amplified from three of five corneal, two of six conjunctival, and none of the iris or capsule samples. The impression cytology samples demonstrated a decline in defensin expression over the three time points studied. There were no false positive results from either the no-RT or negative control samples.

Conclusions-This preliminary study confirms that natural antibacterial peptides are expressed in the anterior segment of the eye. There appears to be a pattern to the expression with inducible $\beta$ defensin 2 not expressed intraocularly and higher levels of $\beta$ defensin 1 than $\beta$ defensin 2 expressed in extraocular tissue. The implication is that $\beta$ defensin 1 is constitutively produced in ocular tissues and represents a key component of the innate immune system.

(Br f Ophthalmol 2000;84:523-526)
\end{abstract}

Defensins $^{1}$ are a family of highly conserved cysteine rich cationic proteins, which have an important role in combating microbial infection. They are found throughout nature, in plants as well as animals and have broad spectrum activity against Gram positive and Gram negative bacteria, as well as enveloped viruses and fungi. Defensins are attracted by the microbial membrane's negative charge and cause disruption by first binding, then intercalating, to form pores that destroy the integrity of the lipid bilayer. This dissipates the mem- brane potential and kills the cell. Defensins have a synergistic action with known antibacterial components of aqueous humour and the precorneal tear film, such as lysozyme and lactoferrin, as well as with antibiotics. As defensins rely on the physical properties of the bacterial cell envelope, they may have an increasingly important role as antibiotic resistance becomes more prevalent.

Two groups of defensins are recognised in humans: $\alpha$ defensins, produced by neutrophils and the Paneth cells of the small intestine, and $\beta$ defensins secreted by epithelial, or epithelial derived tissues. The $\beta$ defensin production at mucosal surfaces is an important part of the innate immune system. Two human $\beta$ defensins have been described, ${ }^{2}$ human $\beta$ defensin 1 (hBD-1) and human $\beta$ defensin 2 (hBD-2).

Eye tissue samples were collected from the anterior segment and used to assay for $\beta$ defensin mRNA production. We hypothesised that the highest $\beta$ defensin expression would be found in tissue with the greatest environmental exposure and that surgically induced inflammation would increase expression.

Quantitation of $\beta$ defensin mRNA was performed with a fluorescent polymerase chain reaction (PCR) (Taqman system). ${ }^{3}$ Briefly, this uses a probe that hybridises to a target sequence within the PCR product. The probe is labelled 5' with a fluorescent reporter and 3' with a quencher dye. The proximity of the two dyes results in no overall fluorescence owing to a Förster type energy transfer. ${ }^{4}$ During the extension step of the PCR, Taq polymerase cleaves the probe separating the reporter dye from the effect of the quencher causing an increase in fluorescence. The increase in fluorescence is used to determine the progress of the reaction and is expressed as the change in fluorescence $(\Delta R n)$ (relative to a passive reference dye) against the number of completed PCR cycles. A positive sample generates an increase in fluorescence that exceeds the threshold value (calculated by taking a point that is $10 \mathrm{SD}$ greater than the average baseline between cycles 3-12), and the cycle number at which this occurs is termed the threshold cycle $\left(\mathrm{C}_{\mathrm{T}}\right)$. By using known amounts of target DNA, a standard curve can be constructed that will give a range of $\mathrm{C}_{\mathrm{T}}$ values for different starting gene copy numbers. From this graph the number of copies of $\beta$ defensin cDNA present in tissue samples can be determined. 
Methods

PATIENTS

Thirty three patients undergoing ocular surgery were recruited to this study. Corneal epithelium, lens capsule, iris, and conjunctiva were collected from a total of 23 patients undergoing phototherapeutic keratectomy (PTK) (five), phacoemulsification (six), and trabeculectomy (12). Corneal epithelium removed before PTK as well as lens epithelium from patients undergoing cataract surgery and peripheral iridectomy specimens from six patients undergoing trabeculectomy were retained for analysis. Conjunctival biopsies $(\sim 0.5$ $\mathrm{mm}$ wide) were collected from a further six patients undergoing trabeculectomy. Impression cytology ${ }^{5}$ (Millipore Corp, Bedford, MA, USA) was performed on a separate group of 10 patients in order to determine changes in the level of defensin expression in response to corneal and conjunctival surgery. These samples were obtained from the central corneal epithelium of six patients undergoing corneal tunnel phacoemulsification at the start of surgery, 3 hours, and 18 hours postoperatively. Conjunctival impression cytology was performed at the same time points on four patients undergoing a trabeculectomy with samples taken $\sim 60$ degrees away from the site of the superiorly placed conjunctival flap. The study followed the tenets of the Declaration of Helsinki and had been approved by the local ethical committee. Informed consent was obtained from all patients after explanation of the nature and possible consequences of the study.

\section{SPECIMEN PREPARATION}

All samples were processed in an identical manner using standard laboratory techniques. In order to denature ribonucleases and preserve mRNA, both the tissue and impression cytology cellulose acetate disc samples were immediately transferred to tubes containing Trizol reagent (Gibco-BRL, UK) and stored at $-70^{\circ} \mathrm{C}$ until the RNA was extracted. After homogenisation of the samples (and the Trizol in which they had been stored) in fresh Trizol, RNA was extracted with chloroform and precipitated by isopropanol. RNA quantification was performed using RiboGreen assay kit (Molecular Probes, Netherlands). The RNA underwent a DNAse incubation (Gibco-BRL, UK) to remove any contaminating genomic DNA, before reverse transcription using the following methods. Primers were annealed to the RNA sequences by heating a mixture of 18 $\mu \mathrm{l}$ of sample with $2 \mu \mathrm{l}$ of a $100 \mu \mathrm{g}$ mixture of random hexamer primers (Promega, UK) at $70^{\circ} \mathrm{C}$ for 10 minutes and then snap cooling on ice for 5 minutes. A $20 \mu \mathrm{l}$ mixture containing the RNA dependent DNA polymerase (Superscript, Life Technologies, UK), buffer, bovine serum albumin, DTT (Life Technologies, UK), dNTPs (Promega, UK), and Rnasin (Rnase inhibitor) (Promega, UK) was incubated for 1 hour at $37^{\circ} \mathrm{C}$. At the end of the incubation the RNA dependent DNA polymerase was inactivated by heating to $95^{\circ} \mathrm{C}$ for 5 minutes.
Table 1 Nucleotide sequence of defensin PCR primers and probes

\begin{tabular}{llllll}
\hline Gene & $\begin{array}{l}\text { Accession } \\
\text { number }\end{array}$ & $\begin{array}{l}\text { Forward } \\
\text { primert }\end{array}$ & $\begin{array}{l}\text { Reverse } \\
\text { primert }\end{array}$ & Probet & $\begin{array}{l}\text { Product } \\
\text { length (bp) }\end{array}$ \\
\hline GAPDH & M33197 & $64-73$ & $272-293$ & $172-209$ & 229 \\
hBD-1 & $\mathrm{U} 73945$ & $86-112$ & $193-172$ & $118-148$ & 86 \\
hBD-2 & Z71389 & $10-27$ & $171-143$ & $106-140$ & 133
\end{tabular}

$\star^{\star}$ Refers to the accession numbers on the EMBL database. †The numbers refer to the coordinates of the sequences on the EMBL database.

PCR PRIMERS AND METHODS

Real time semiquantitative PCR was performed using non-fluorescent primers and a double labelled internal probe. The probe was labelled 5' with FAM (6-carboxy-fluorescein) and 3' with TAMRA (6-carboxy-tetramehylrhodamine), that acted as a fluorescence quencher. The PCR was performed on a 7700 sequence detector (ABI Prism, USA) with primers (Table 1) designed from published sequences for glyceraldehyde-3-phosphate dehydogenase (GAPDH), hBD-1, and hBD-2. To confirm the specificity of the assay the PCR product was purified (Geneclean kit, BIO 101, CA, USA) and sequenced (Sequenase, Amersham).

DNA amplification was performed in $25 \mu 1$ reaction volume, containing cDNA, $3.5 \mathrm{mM}$ $\mathrm{MgCL}_{2}, 2.5 \mu \mathrm{l}$ of reaction buffer, $200 \mu \mathrm{M}$ dNTPs, $300 \mathrm{nM}$ forward and reverse primers, $100 \mathrm{nM}$ of probe, $0.25 \mathrm{U}$ of Amp Erase (Uracil n-Glycosylase), and $0.625 \mathrm{U}$ of Ampli Taq Gold in the final reaction mix (all reagents Perkin Elmer, UK). The thermal cycling conditions were an initial 10 minutes at $94^{\circ} \mathrm{C}$, followed by 50 cycles of denaturing at $94^{\circ} \mathrm{C}$ for 15 seconds, with combined annealing and extension at $60^{\circ} \mathrm{C}$ for 60 seconds. To confirm the elimination of genomic DNA contamination, a "no reverse transcription" (RT) control was included for all samples. Standard curves for hBD-1 and 2, correlating $\Delta \mathrm{Rn}$ with starting gene copy numbers, were generated using separate plasmids incorporating these genes: this enabled the starting template copy numbers for each sample to be determined. To take into account variation in the size of the samples $\beta$ defensin mRNA concentrations are expressed as a ratio to the levels of the housekeeping gene (GAPDH). This enabled $\beta$ defensin expression per unit tissue to be calculated for every sample.

\section{Results}

Direct sequencing of representative PCR amplicons extracted from the gel in the preliminary study confirmed the primer's ability to amplify hBD-1 and hBD-2 DNA (results not shown). A positive PCR result, demonstrated by an increase in fluorescence above threshold, indicated that hBD-1 and hBD-2 were amplified from 21 and five of the 23 tissue specimens respectively. hBD-2 was mainly expressed at lower levels than hBD-1 and was present only in specimens derived from the ocular surface (Table 2). Since the sensitivity of semiquantitative PCR declines at very low levels of template the mean hBD-1 level in the tissue samples was calculated from the 20 
Table 2 Tissue sample qualitative and semiquantitative PCR results obtained from the 23 tissue samples

\begin{tabular}{lllll}
\hline Sample type & $h B D-1^{*}$ & $h B D-2^{*}$ & $\begin{array}{l}h B D-1 / G A P D H+ \\
\text { Mean }(S E M)(S D)\end{array}$ & $\begin{array}{l}h B D-2 / G A P D H+ \\
\text { Mean }(S E M)(S D)\end{array}$ \\
\hline Cornea & $5 / 5$ & $3 / 5$ & $0.166(0.084)(0.187)$ & $0.353(0.292)(0.653)$ \\
Conjunctiva & $5 / 6$ & $2 / 6$ & $0.568(0.290)(0.709)$ & $0.013(-)(0.018)$ \\
Iris & $5 / 6$ & $0 / 6$ & $0.232(0.116)(0.285)$ & - \\
Lens capsule & $6 / 6$ & $0 / 6$ & $0.093(0.056)(0.137)$ & - \\
\hline
\end{tabular}

${ }^{\star}$ Refers to the number of samples from which the two human $\beta$ defensins were amplified. †Refers to the human $\beta$ defensin mRNA levels expressed as a ratio to the levels of the housekeeping gene (GAPDH).

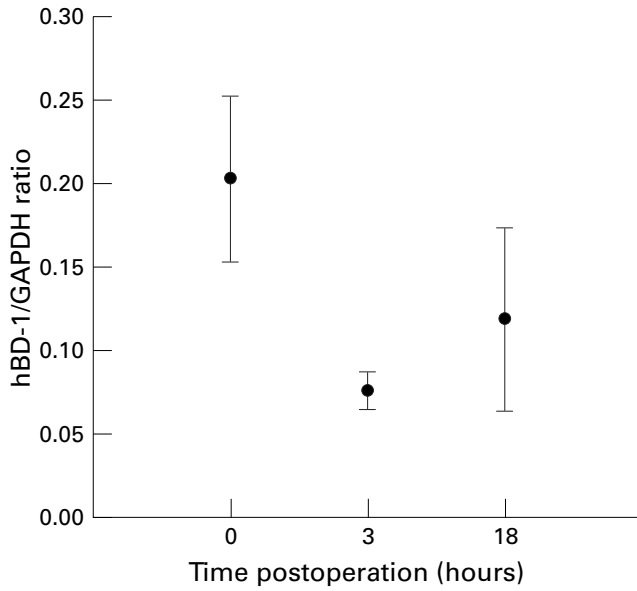

Figure 1 Graph illustrating the ratio of $h B D-1$ and GAPDH gene expression in conjunctival impression cytology specimens postoperatively. The error bars denote the standard deviation of the measurements.
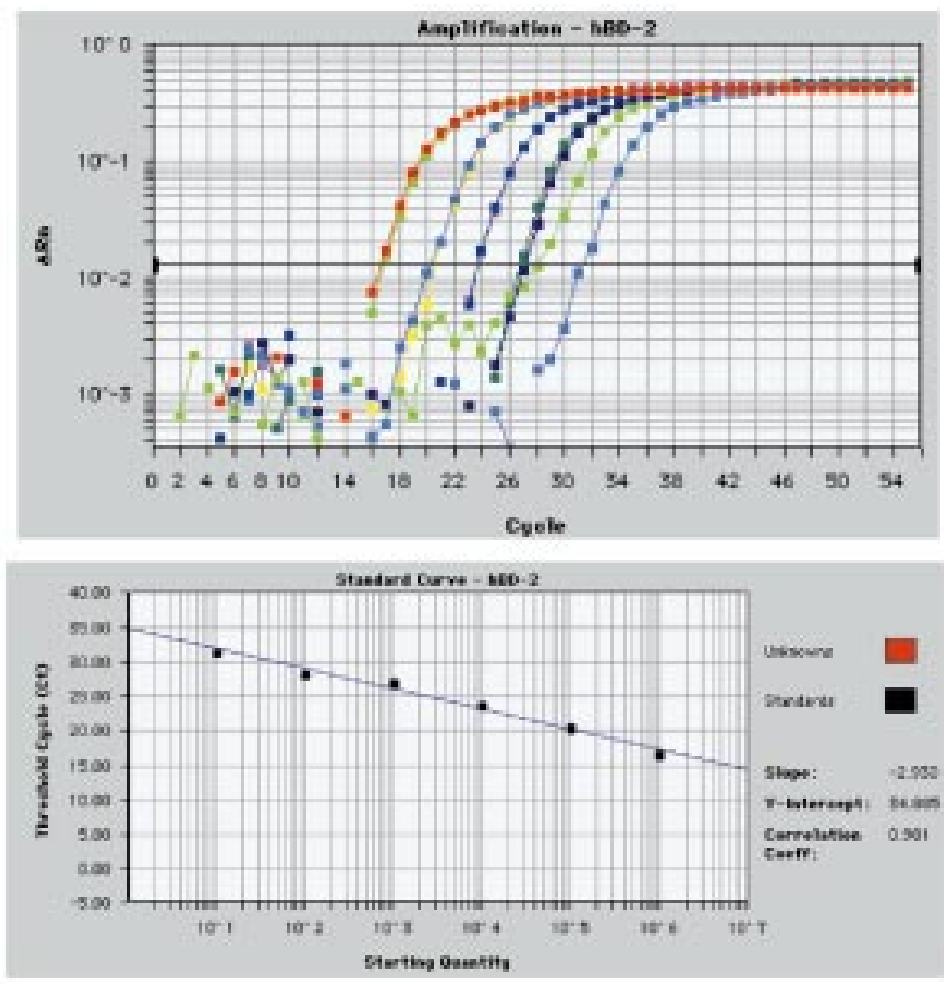

Figure 2 The upper graph plots the change in fluorescence ( $\triangle R n$ ) as the PCR reaction proceeds against the number of completed PCR cycles; the increase in fluorescence accurately mirrors the rise in PCR product ( $h B D-2)$. Using known amounts of target DNA, individual curves are generated for $\triangle R n$ and the number of PCR cycles required for an increase in fluorescence that exceeds the threshold value $\left(\triangle R n=\sim 10^{-2}\right.$, denoted by the horizontal line) is measured. The points, where each curve exceeds the threshold value, are used to construct the standard curve shown in the lower graph. By performing semiquantitative PCR on samples containing unknown amounts of defensin DNA and measuring the number of PCR cycles required for $\triangle R n$ to exceed the threshold value, the amount of $\beta$ defensin present can be determined by extrapolating from the standard curve. Standard curves for $h B D-1$ and GAPDH were generated in an identical manner. samples with PCR results more than 10 copy numbers (Table 2).

A decline in hBD-1 expression was observed in the conjunctival impression cytology specimens over the time points studied (Fig 1), although this change was not statistically significant $(\mathrm{p}=0.34$, independent $t$ test and $\mathrm{p}=0.083$, non-parametric testing). hBD-2 mRNA was detected in three out of the four samples taken at the time of surgery, but became undetectable in two out of the four samples 3 hours postoperatively, and in three out of four samples at 18 hours postoperatively. The small numbers in this study precluded statistical analysis of the data. The levels of hBD- 1 and 2 expression in the corneal impression cytology specimens were at the lower limit of the PCR's sensitivity preventing quantitative analysis of temporal patterns in defensin expression.

\section{Conclusions}

The first reports of ocular defensin expression demonstrated with non-quantitative $\mathrm{PCR}^{67}$ that defensin mRNA is expressed on the ocular surface. Using a semiquantitative assay to measure the level of expression (Fig 2) we have confirmed these findings, demonstrated that $\beta$ defensins are also expressed intraocularly and provided novel information about the pattern of $\beta$ defensin expression in ocular tissues.

We have presented evidence that hBD-1 mRNA is expressed both intraocularly and extraocularly while hBD-2 expression is confined to the ocular surface. hBD-1 was detected more frequently and generally at higher levels than hBD-2 in both the tissue and impression cytology specimens. The overall pattern of defensin expression suggests that the two $\beta$ defensins have different roles in the eye's innate immune response with hBD-1 serving as a defensive mechanism in the absence of inflammation. It is probable that the eye behaves like other mucosal surfaces where $\mathrm{hBD}-2$ is inducible and inflammation increases its expression. ${ }^{8}$

The semiquantitative RT-PCR assay used offers certain advantages over qualitative tests, in particular the ability to measure directly, and in real time, the level of expression. In the future this may enable the factors that control defensin expression in vivo to be investigated. Combining the assay with impression cytology allows multiple ocular surface samples from the same patient to be analysed. Interpretation of our impression cytology results, which provide evidence of a decline in conjunctival hBD-1 expression in the immediate postoperative period, was limited by the assay's sensitivity and the small number of patients in this part of the study. Techniques to increase the PCR's sensitivity will need to be developed and the use of an additional extraction procedure, such as QIAmp Tissue Kit (Qiagen) that allows the separation of sample contaminants, would help prevent inhibitory material in clinical samples being carried over into the PCR decreasing sensitivity. Use of larger diameter impression cytology cellulose acetate discs may also play a 
part as these would double the surface area sampled increasing the number of cells and hence mRNA collected.

The ability to measure defensin expression accurately may provide molecular explanations for the mechanisms underlying ocular surface and intraocular infections. For instance the antibacterial properties of aqueous (in the absence of prophylactic antibiotics) are sufficient to eliminate the bacterial contamination of the anterior chamber that occurs in up to a third of cataract operations. ${ }^{9}{ }^{10}$ The demonstration of hBD-1 expression in uninflammed intraocular tissue suggests that this peptide may be partly responsible for the observed antimicrobial effect, as has been previously postulated. ${ }^{11}$ Another example is late endophthalmitis following trabeculectomy, which is associated with the use of antimetabolites that profoundly alter the morphology of the conjunctival bleb. Using the assay described to investigate whether alterations in defensin gene expression are implicated in the increased susceptibility of such blebs to infection, is now a real possibility. In the future, investigation of defensin expression may improve not just our understanding of the pathogenesis of ocular infections but provide us with the means both to intervene therapeutically and in the longer term by upregulating expression, reduce the chance of infection occurring.

Proprietary interest: None.

Supported by grants from the Medical Research Council and the British Lung Foundation. IRH is recipient of British Medical Association HC Roscoe fellowship.

We are grateful to Mr P T Khaw, Mr R A Hitchings, and Mr We are grateful to Mr P T Khaw, Mr R A Hitchin
R Collyer-Powell for their assistance with this study.

1 Hancock REW. Peptide antibiotics. Lancet 1997;349:41822.

2 Diamond G, Bevins CL. $\beta$-defensins: endogenous antibiotics of the innate host defence response. Clin Immunol Immunopathol 1998;88:221-5.

3 Gelfand DH, Holland PM, Saiki RK, et al. US Patent 5210015 1993; Chem Abstract 1992.

4 Lakowicz JR. Principles of fluorescent spectroscopy. New York: Lakowicz JR. Principles of fluorescent spect
Plenum Press, Chapter 10, 303-339.

5 Dart JK. Impression cytology of the ocular surfaceresearch tool or routine clinical investigation? $\mathrm{Br} \mathcal{F}$ Ophthalmol 1997;81:930.

6 Haynes RJ, Tighe PJ, Dua HS. Innate defence of the eye by anti-microbial defensin peptides. Lancet 1998;352:451-2 .

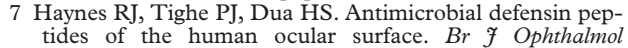
1999;83:737-41.

8 Singh PK, Jia HP, Wiles K, et al. Production of $\beta$-defensins by human airway epithelia. Proc Natl Acad Sci 1998;95: 14961-6.

9 Sherwood DR, Rich WJ, Jacob JS, et al. Bacterial contamination of intraocular and extraocular fluids during contamination of intraocular and extraocular fluids during
extracapsular cataract extraction. Eye 1989;3:308-12.

10 Kattan HM, Flynn HW, Pflugfelder SC, et al. Nosocomial endophthalmitis survey: incidence of infection after intraocular surgery. Ophthalmology 1991;98:227-38.

11 Diamond JP, Leeming JP, Smart AD, et al. An antimicrobial effect associated with rabbit primary aqueous humour. $\mathrm{Br} \mathcal{F}$ Ophthalmol 1994;78:142-8. 